\title{
A method for assessing the stability of digital automatic control systems (ACS) with discrete elements. Hypothesis and simulation results
}

\author{
Kodkin Vladimir ${ }^{1}$, Baldenkov Alexander ${ }^{2}$ and Anikin Alexander ${ }^{3}$ \\ 1 South Ural State University, Chelyabinsk, Russia; kodkina2@mail.ru \\ 2 South Ural State University, Chelyabinsk, Russia; baloo@mail.ru \\ 3 South Ural State University, Chelyabinsk, Russia; anikinas@susu.ru
}

\begin{abstract}
The article presents a new approach to the analysis of the stability of automatic systems with discrete links.

In almost all modern automatic control systems, there are links that break signals in time. These are power controlled switches - transistors or thyristors operating in a pulsed mode and digital links in regulators.

Time discretization significantly affects the stability of processes in the automatic control system. The theoretical analysis of such systems is rather complicated and requires a significant change in engineering approaches to analysis. In connection with the improvement of digital controllers and a significant increase in their performance, in recent years this problem has practically not been remembered. However, its mathematical "content" has not changed since the 80 s of the 20th century, when discreteness began to play a major role among the problems hindering progress in automatic control systems, in terms of the transition to digital systems.

In this paper, a new approach is proposed, which consists in interpreting the sampling operation by a link with the proposed frequency characteristic, which determines the suppression of input high-frequency signals. This link greatly simplifies engineering calculations and demonstrates the new capabilities of sampling systems. These possibilities include the rational distribution of digitalization resources - the number of bits and the sampling interval between the regulator channels, depending on the frequency range of the efficiency of these channels. Theoretical statements have been verified and confirmed by simulation. It is shown how this approach makes it possible to formulate new principles of construction of seemingly well-known controllers - PID controllers and variable structure systems (VSS).
\end{abstract}

Keywords: discreteness, frequency response, automatic control system, PID controller, variable structure system with sliding processes

\section{Introduction}

Discreteness of signals "appeared" in the ACS as a result of the technological improvement of its elements - regulators and power electronics. In power electronics, impulse control has made it possible to significantly increase the efficiency of converters, and in control systems, digital technologies have a number of advantages over analog technologies, about which a huge number of works have been written and which no longer raises any doubts. Discreteness, in this case, is perceived as an inevitable problem, which, however, is solved when the sampling rate is increased to such a level at which discreteness is no longer a problem for electromechanics.

If the sampling frequency in the ACS is a few microseconds, and the switching period of the impulse elements is $5-10 \mathrm{kHz}$, then for electric drives, the main processes in which are within the spectrum of 10-100 Hz, one can "forget" about the unpleasant discreteness. So, at least, one might think based on the attention given to this problem in the work on digital electric drives. However, 
for high-precision electromechanical systems, the problems of discreteness of information signals and power currents remain important. Indeed, the discreteness in time and in the level of the processed signals inevitably breaks the continuous ACS and makes their behavior in terms of the stability of processes unpredictable. This is especially important for systems that are essentially nonlinear, in which it is rather difficult to predict the reaction to all possible variations of the setting signals and disturbing factors.

\section{Problem statement. State of the issue}

All methods of analysis of discrete, impulse, digital systems are somehow connected with the use of delay links and lattice functions [1, 2, 10-15, 16, 17]. These are discrete transformations of continuous signals and transfer functions - Z-transformations, D-transformations, discrete Laplace transforms and others. What they have in common, and the main thing for working with real ACS, is that ALL elements of the control system undergo transformations - continuous, linear, with simple and complex transfer functions. But even then, precise transformations cannot theoretically be carried out.

This is well explained in the classic works on automatic control theory (ACT) of the 80s. For example, in the book by M.V. Meerov "Fundamentals of Automatic Control" (Moscow, "Nedra", 1972) [1] on page 332 says about inverse Z-transformations: "the transformation makes sense if the series converges ...". The calculation results, in the same book: "you can restrict yourself to a finite number of terms of equation (7.137)" and the frequency response of the discretization link is reduced to the characteristic of the delay link:

$$
W^{*}(j \omega) \approx \sum_{n=0}^{N} w_{n} e^{-j \bar{\omega} n}
$$

In this case, it is stipulated that "the clock frequency is greater than the range of frequencies under consideration".

Detailed mathematical calculations in the later, very widely used and in modern works on ACS, book by Ya.Z. Tsypkin "Foundations of the theory of automatic systems" (Moscow, "Nauka", 1977) [2], in paragraphs 25.3 and 25.4 and, finally, in 28.2 it is stated: "... for sufficiently small pulse repetition periods, the impulse system can be considered as continuous, containing the same continuous part and a delay element", those the structures shown in Figure 1 are equivalent.

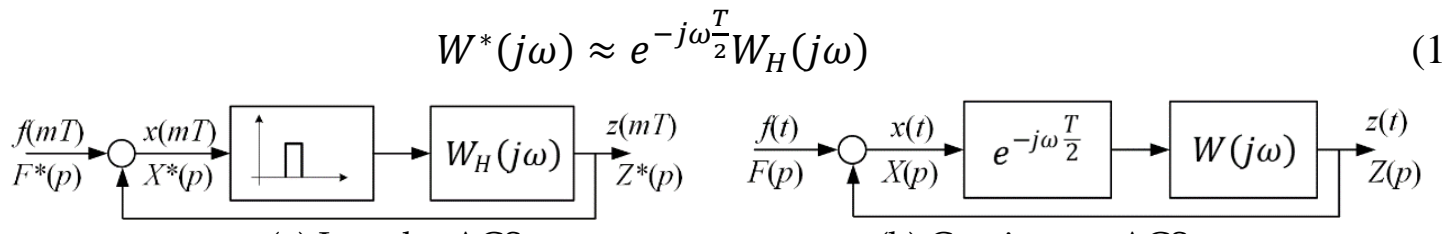

(a) Impulse ACS

(b) Continuous ACS

Figure 1.

At the same time, it is also said about "small" sampling time, although it is not specified how small it should be, and how wrong, if not "small".

For engineers, this condition sounds something like this: "The cutoff frequency of the system must be at least 10 times less than the quantization frequency. Otherwise, nothing can be guaranteed. " In addition, the delay link does not change the amplitude frequency response, i.e., if the condition of the "smallness" of the quantization interval is met, it can be forgotten altogether.

Over the past years, a lot of works on these topics have been written and published, of course, but, in almost all, the approach has remained unchanged. All methods are based on discrete Laplace transforms [10-15]. And all the same assumption about the possibility of "forgetting" about the discreteness of the ACS in the analysis of stability, if the sampling frequency is significantly higher than the frequencies of the spectra of changes of coordinates of interest to the developers of ACS. These coordinates are most often the speed or angular movement. Moreover, the spectrum of signals reflecting changes in these coordinates lie within $10-100 \mathrm{~Hz}$, and change little, while discrete elements in modern ACS have a sampling rate tens of thousands of times higher. The most serious discrete 
element is the power impulse elements. The sampling frequency of pulse elements is $2-10 \mathrm{kHz}$, which is also significantly higher than the frequency of the spectrum of mechanical coordinates, but noticeably less than the frequency of the ACS signals.

\section{Suppression link. Frequency interpretation of signal sampling}

Many years of experience with electromechanical systems, theoretical research and modeling have shown that the traditional representation of the discretization units with delays has two "drawbacks" in the analysis of the stability of the ACS [6, 7].

This is, firstly, the formal possibility of correcting the phase shift in systems with delay, and, secondly, the "non-influence" of such links on the amplitude characteristics. To overcome these "weaknesses" and show the influence of sampling links on the stability of the ACS. It is proposed that a suppression link, the main property of which is the complete suppression of input signals with a frequency higher than or equal to the sampling frequency.

The formula for the frequency response of the suppression link can be as follows:

$$
W=A(\omega) e^{j \varphi(\omega)},
$$

where $A(\omega)$ и $\varphi(\omega)$ - respectively, the amplitude and phase frequency characteristics, and have the following dependences:

$$
\begin{array}{r}
\varphi(\omega)= \begin{cases}-\frac{K_{1} \cdot(\tau \omega)}{1-\omega \tau}, \text { при } \omega \leq \frac{1}{\tau} \\
-\infty, \quad \text { при } \omega>\frac{1}{\tau}\end{cases} \\
A(\omega)= \begin{cases}K_{2} \cdot e^{\frac{1}{\omega \tau-1}}, \text { при } \omega \leq \frac{1}{\tau} \\
0, & \text { при } \omega>\frac{1}{\tau}\end{cases} \\
\operatorname{Lg}[A(\omega)]= \begin{cases}\frac{K_{3}}{\omega \tau-1}, \text { при } \omega<\frac{1}{\tau} \\
-\infty, & \text { при } \omega>\frac{1}{\tau}\end{cases}
\end{array}
$$

Graphical interpretation of the frequency response of the suppression link is shown in Figure 2.
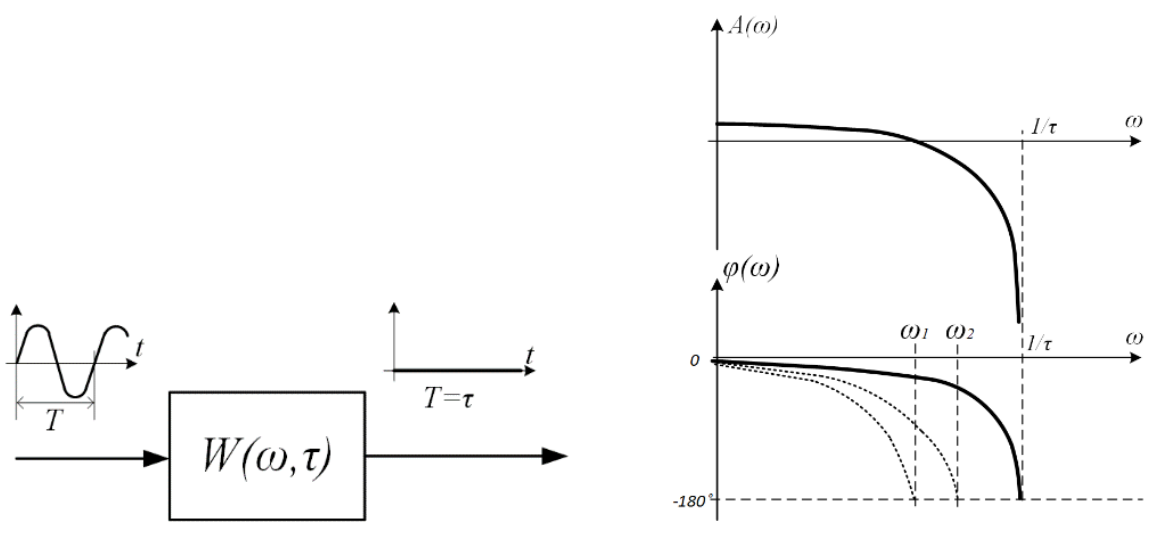

Figure 2.

Formulas $(2,3,4)$ differ from formula (1), especially in the frequency zone close to the sampling frequency $\omega=\frac{1}{\tau^{\prime}}$ in this frequency zone, the signal is suppressed, which cannot be overcome by sequential correction, since no serial link will be able to overcome the suppression of the amplitude expressed by the formula (3). 
The phase shift (formula 2), at low frequencies similar to the shift of the delay link, in the frequency zone $\omega=\frac{1}{\tau}$ increases sharply in absolute value and also cannot be corrected in the frequency zone close to the sampling frequency.

As follows from the formulas and graphs of the frequency characteristics of the proposed suppression link, with any sequential correction at a frequency below the quantization frequency, the phase shift will reach a critical value (- 180 degrees) and lead to the instability of a closed loop according to the Nyquist criteria [3] for linear ACS or V. M. Popov [5, 9] for nonlinear systems. How far from the quantization frequency $\left(\omega=\frac{1}{\tau}\right)$ this will happen depends on other links of the system.

Analytically, it is rather difficult to confirm the adequacy of such a representation of signal sampling operations in time, while the graphical interpretation seems to be quite clear. Modeling can provide even more clear confirmation of the effectiveness of introducing such links into the ACS to analyze the effect of signal discreteness on the ACS stability, even if they are not rigorously interpreted.

In the 15th chapter of the book [18], several CAP models are considered. These examples can be used to assess the capabilities of the "new" approach in comparison with the traditional methods of analyzing the stability of ACS. "New", both in relation to the sampling operation itself, and to the CAP as a whole.

Consider the stability conditions for the second-order ACS model with proportional - integral differential controllers (PID controllers), and variable structure systems with sliding modes (VSS with SM).

\section{ACS with PID controller. Frequency response analysis and simulation}

The PID controller is the most widely used type of controller in industrial automation. This regulator has three links, each with its own purpose and frequency range of "work":

- proportional channel $(\mathrm{P})$ - the channel is responsible for the speed of the system and for the overall dynamics of the control loop; it is effective in the zone of the control loop cutoff frequency;

- differential channel (D) - the channel ensures the stability of the system and, according to the Nyquist criterion [3] and its engineering interpretations, should operate in the range from the cutoff frequency to a frequency 10 times higher;

- integrator (I) - provides high static accuracy of the control system, it is necessary in the zone of low frequencies, the range of its action should be less than the cutoff frequency by about 10 times, according to the same engineering stability conditions.

PID controller transfer function:

$$
W=K+T_{i} p+\frac{1}{T_{d} p} .
$$

In a continuous ACS of this kind, a loop with a zero static error is easily synthesized, and the time of the transient process is 10-15 times less than the time constant of the control object.

So, for the model, the diagram of which is shown in Figure 3a, the transients are shown in Figure $3 b$.
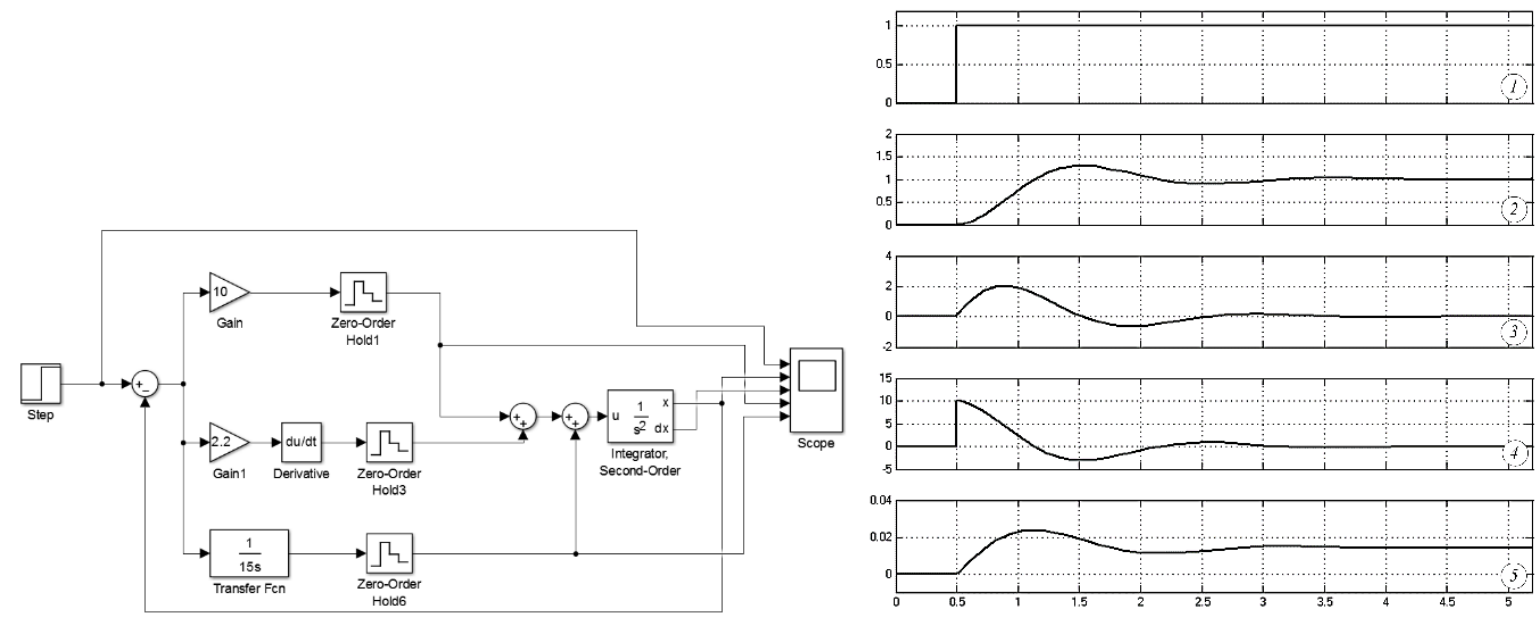
(a)

(b)

Figure 3.

The control object is represented by a double integrator with an integration constant of $1 \mathrm{~s}$. Here "Gain" is a proportional gain channel with $\mathrm{K}=10$; "Derivative" is a differentiating channel with a time constant of 2,2s; "Transfer Fcn" is an integrating channel with a time constant of 15s.

As follows from the diagrams in Fig. 3b, the stability of the processes is close to the boundary, the time of the transient process is 1,3-1,5s. Here: 1 is the reference signal, 2 is the controlled value, the 3 its derivative, 4 and 5 are the outputs of the regulator channels - the proportional channel and the integrator, respectively.

The links in the PID controller are connected in parallel, their resulting frequency characteristics can be determined by the rule "on top", Figure 4a shows the logarithmic frequency characteristics (LFC) of the continuous PID controller.

When "adding" the "Zero order Hold" sampling links to each channel of the regulator, changes occur both in the LFC and in the processes in Figures $5 a$ and $5 b$.

As follows from the frequency characteristics in Figure $4 \mathrm{~b}$, the sampling frequency of the Integral and Proportional channels does not affect the resulting LFC of the regulator if these frequencies are higher than $\omega_{1}=\frac{1}{K \cdot T_{i}}$ and $\omega_{2}=\frac{K}{T_{d}}$, respectively.

Only the quantization frequency of the differential channel affects the differentiating properties of the regulator - its stabilizing "abilities". When it decreases (Fig. 4c) below $\omega_{2}$, the differentiating properties of the regulator deteriorate significantly.

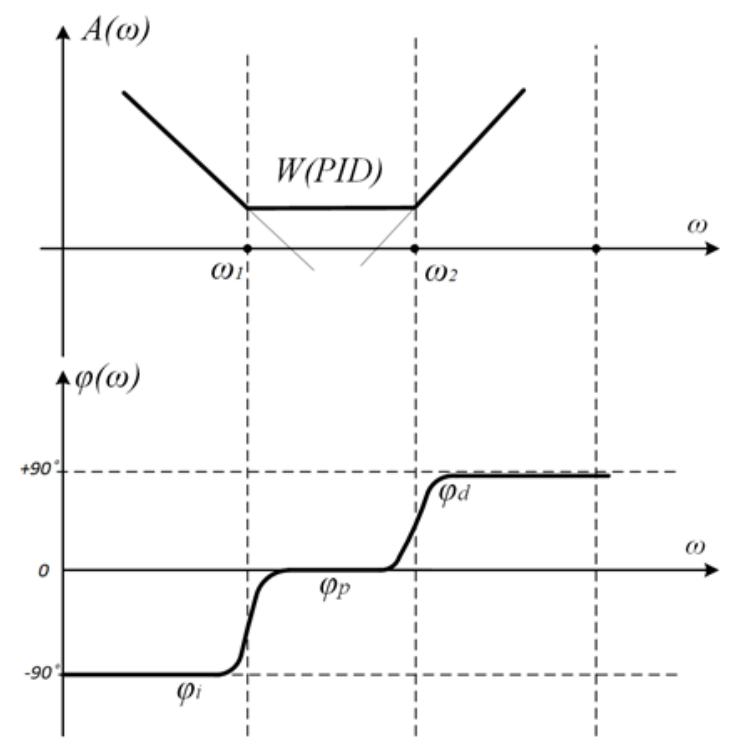

(a)

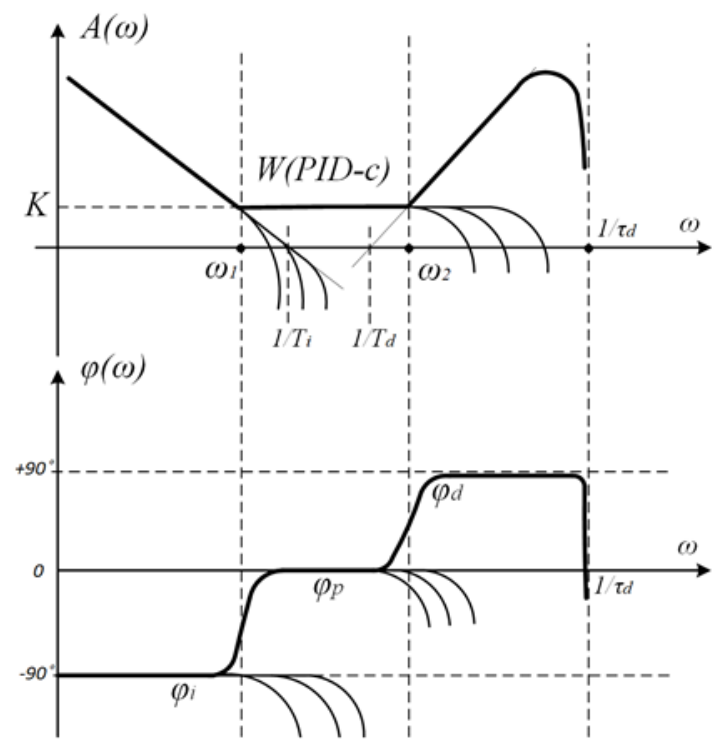

(b)

Figure 4 .

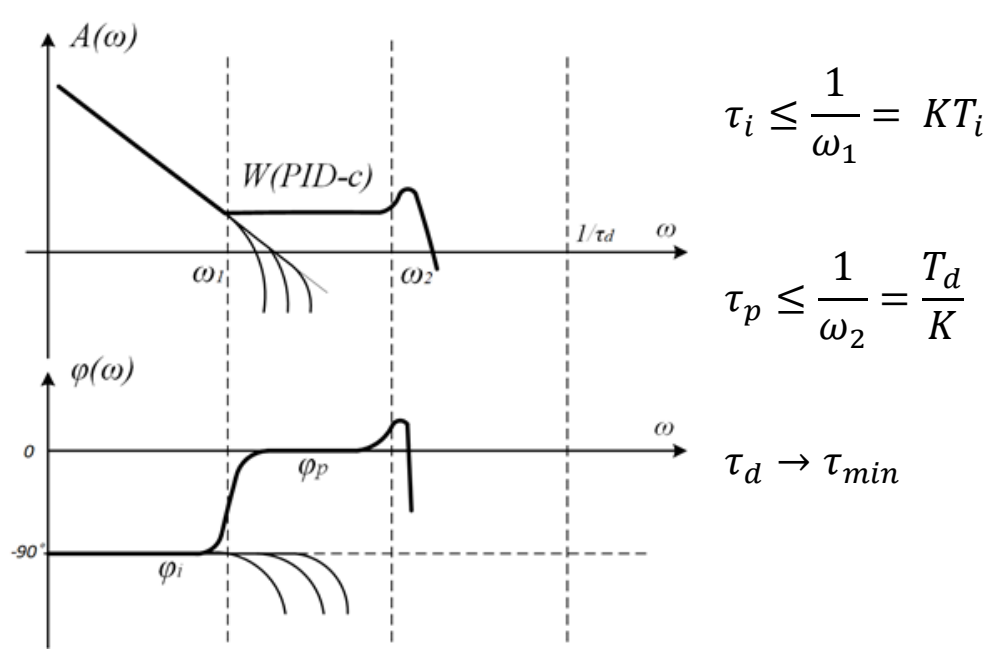


(c)

\section{Figure 4.}

Modeling fully confirmed these assumptions. Figures $5 \mathrm{a}$ and $5 \mathrm{~b}$ show the processes in the model.

Usually, stability in such systems is analyzed by engineering interpretations of the Nyquist criterion and is determined by the phase shift of the frequency response of the equivalent link at the cutoff frequency of the closed loop, which determines the speed of the system [3].

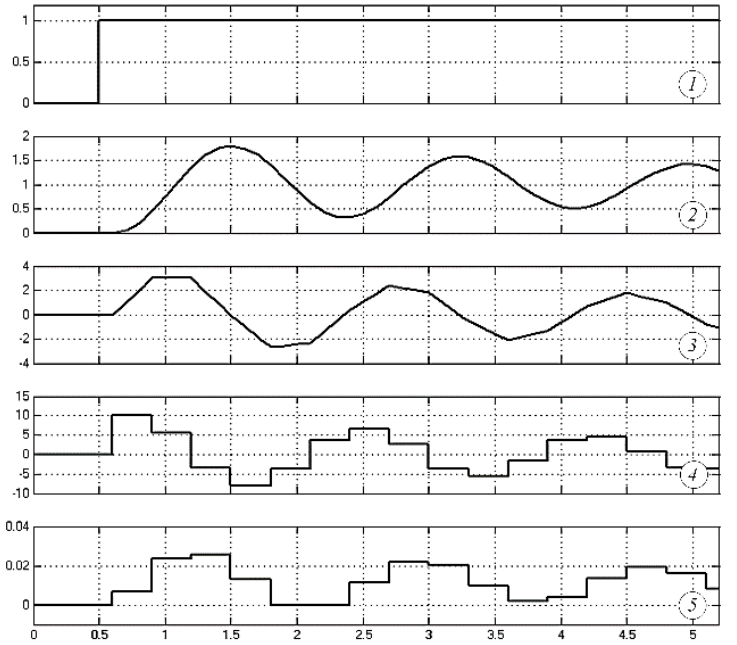

(a)
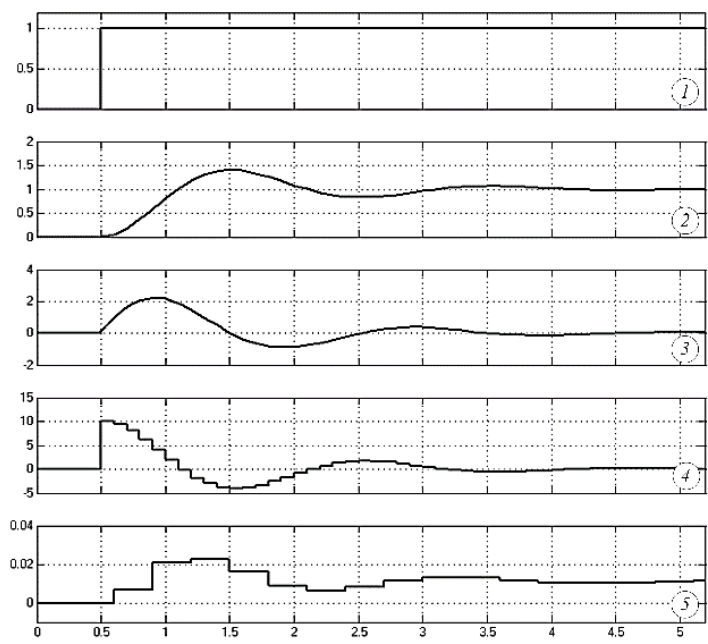

(b)

Figure 5.

With an increase in the quantization time to $0,3 \mathrm{~s}$ in all channels, the processes became oscillatory (Fig. 5a), since The PID controller became a PI controller, the LFC are shown in Figure 4c, and the processes in the model are shown in Figure 5a (diagrams: 1-reference signal, 2-controlled variable, 3-derivative of the controlled variable, 4-output signal of the differentiating channel of the controller after sampling, 5-output signal of the integrator).

Further, in the differential channel, the discreteness is significantly reduced, to $0,1 \mathrm{~s}$ (graph 4 in Figure $5 b$ ), and in other channels this discreteness remained the same $-0,3 \mathrm{~s}$ (graph 5 in Figure $5 b$ ).

As follows from the LFC of the regulator with discreteness links and processes in the model, with a high speed of the channel for differentiating the discreteness of the proportional and integral channels, they practically do not affect the stability of the ACS.

From the simulation results, in addition to the conclusion about the validity of the proposed interpretation of the suppression link and the confirmation of the possibility of using engineering variants of the stability criteria of Nyquist and V.M. Popov, a very useful practical conclusion follows.

In the PID controller, you can organize a low sampling rate in integral channel, where high accuracy is required, i.e. many bits, but fast quantization in a differential channel where high accuracy is not required, i.e. the number of discharges can be reduced.

This result can be called efficient multifrequency sampling. Naturally, it would be simply impossible to obtain such a solution with the traditional approach.

\section{An example with variable structure systems (VSS)}

It is of interest how the transfer function of the suppression link will "manifest" itself in systems with a variable structure with sliding processes. Figure 6 shows a block diagram of the VSS with a sliding mode (SM). Here $\mathrm{CO}$ is a control object (second-order integrator) with a Wco transfer function, TG is a switching path generator ("slip" - $\left.\mathrm{W}_{\mathrm{TG}}\right), \mathrm{C}$ is an amplifier $\left(\mathrm{W}_{\mathrm{C}}\right)$. 


\section{Figure 6.}

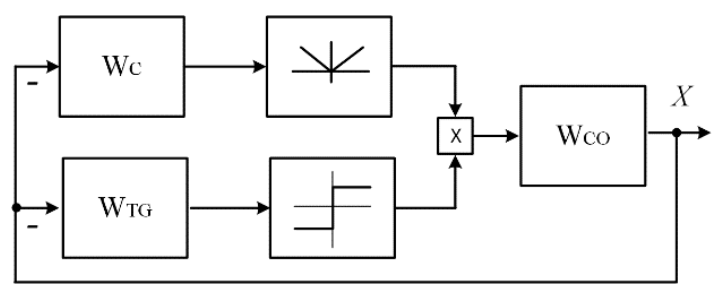

As is known [4, 8], in such systems the stability condition for sliding processes is divided into the conditions for the existence of "slow" processes (stability condition) and the conditions for ideal sliding, that is, the existence of fast motions around slow trajectories with infinitely high frequency and infinitesimal amplitude. "Real" fast movements are usually found in real ACS. Discretization can affect the fulfillment of both slip conditions. The frequency characteristics of the proposed link turn out to be a very convenient tool for frequency slip conditions. In [8], a frequency condition for the existence of sliding processes was obtained. To use this condition, the concept of an equivalent circuit is introduced in Figure 7. In this circuit, the same links are connected in a slightly different way.

Figure 7.

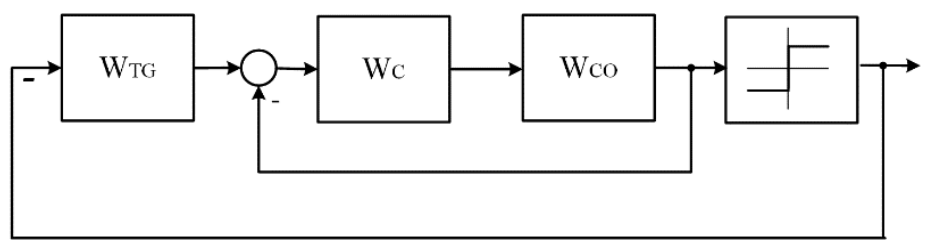

Slip condition according to the frequency characteristics of the ACS links:

The condition of ideal sliding is met when two elements - the sliding trajectory generator and the circuit formed by the controller and controlled member - are connected in series with equivalent phase characteristic of $-90^{\circ}$ minimum, and the value of $-90^{\circ}$ is reached at $\omega \rightarrow \infty$.

The suggested frequency condition is met if the real part of frequency characteristics under consideration transferred to the complex space is positive, or in a slightly different formulation:

$$
\text { if } W_{K}=\frac{W_{C} \cdot W_{C O}}{1+W_{C} \cdot W_{C O}},
$$

then for sliding it is necessary and sufficient that the condition is fulfilled for all frequencies:

$$
\begin{aligned}
& \operatorname{Re}\left[W_{K} \cdot W_{T G}\right]>0 \\
& \varphi\left[W_{K} \cdot W_{T G}\right]>-90^{\circ}
\end{aligned}
$$

If this condition is not met at frequencies close to the spectrum of "slow" movements in the VSS, then the system is unstable, if in the frequency region 10 or more times higher, then "fast" sliding movements are not ideal, that is, they have a finite frequency and amplitude.

The diagram of the model is shown in Figure 8. Here the amplifying channel is represented by the sum of the integrating channel and the proportional link with a time constant of 10 s and a gain of 100 , the sliding path is formed as a first order forcing link with a time constant of 0,15 s ("Derivative" link), object control, as in the model with a PID controller - a second-order integrator with a time constant of $1 \mathrm{~s}$. 


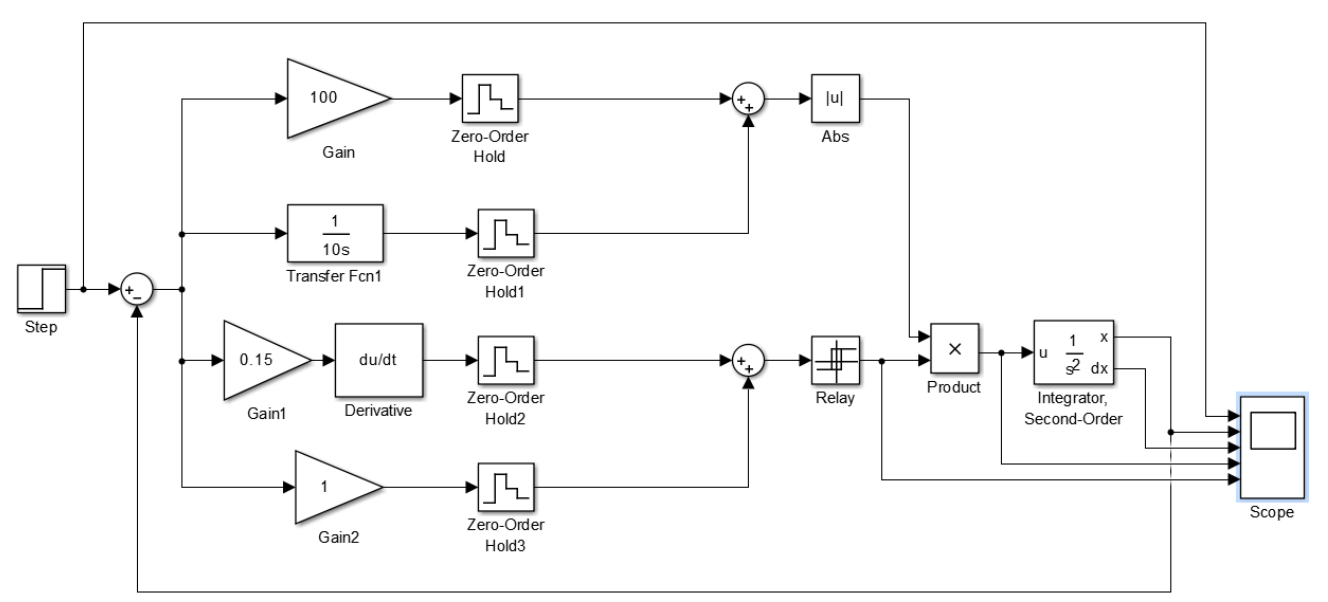

Figure 8.

Figure 9a shows the frequency response for continuous channels of the regulator. Here: $\omega_{1}$ is the conjugation of the characteristics of the integrator and the proportional link, $\omega_{2}$ is the conjugation of the characteristics of the differentiating channel and the proportional one in the trajectory shaper, $\omega_{\mathrm{c}}$ is the cutoff frequency of the slow motion contour in the VSS, Figure 9b shows the processes when all slip conditions are met. Here: 1-reference signal, 2-adjustable value, 3-derivative, 4-signal at the output of the slip driver, 5-output of the relay element "Relay".

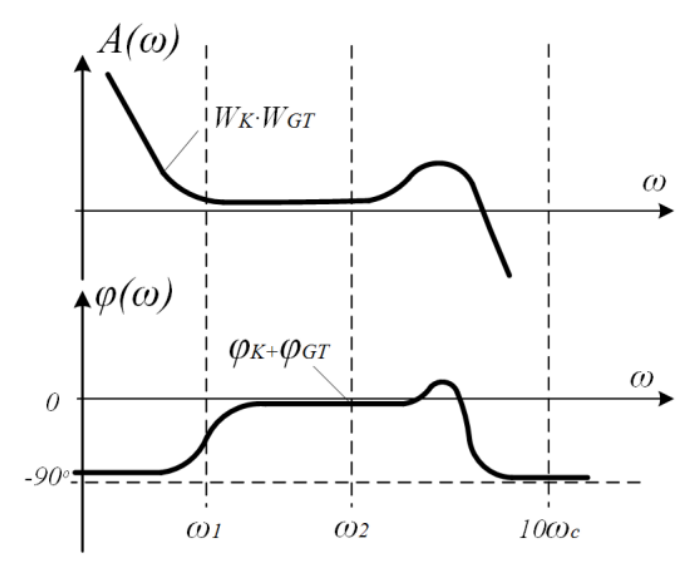

(a)
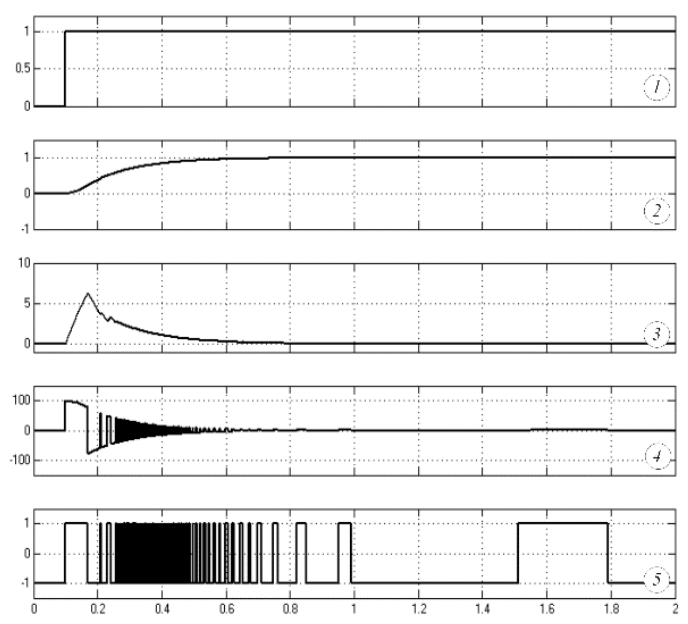

(b)

Figure 9.

With the introduction of discrete links and corresponding suppression links into the model circuit, slip conditions and processes change. In Figures 10 and 11 - 1-reference signal, 2-adjustable value, 3-derivative, 4-derivative signal after a discrete link, 5-relay element output.

If the condition is violated in the high-frequency zone, this is a real slip, the frequency characteristics are shown in Figure 10a, the processes - in Figure 10b. This violation is influenced by the discreteness of the differentiating channel of the slip driver and the proportional channel of the amplifier. This difference from a system with a PID controller is explained by the fact that in the ACS the stabilization and gain channels are not added, as in linear controllers, but are multiplied, i.e. work in parallel. But the discreteness of the integral channel also does not affect the processes if the simple condition $\tau_{i} \leq \frac{T_{i}}{K}$ is satisfied. In this model, the parameters of the sampling units are as follows: amplification units - proportional unit - 0,3s, integrator - $0,5 \mathrm{~s}$, in the trajectory shaper, the sampling interval of the differential unit is $0,01 \mathrm{~s}$ 


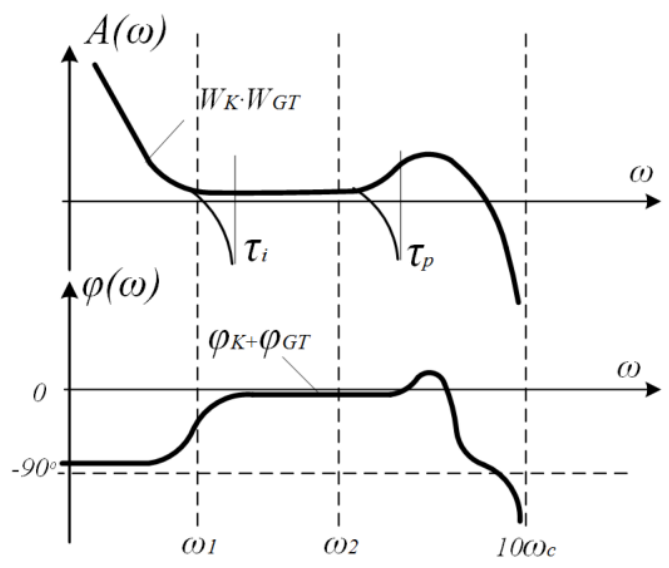

(a)
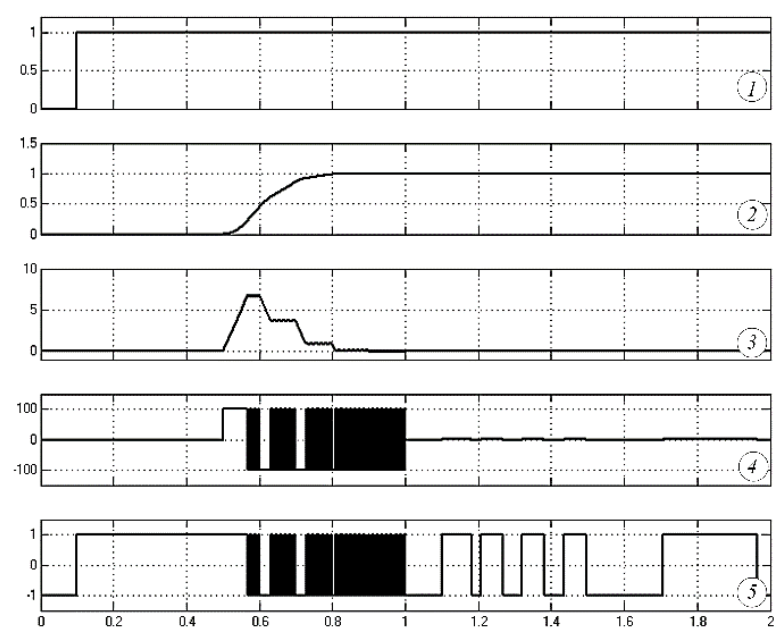

(b)

Figure 10.

If slip conditions (6), (7) are violated in the low-frequency zone, then slip does not exist - the characteristics are shown in Figure 11a, processes in Figure 11b. Such a state is formed in the system if the sampling interval of the differentiating channel is greater than 0,15s. For the VSS in Figure 11, the parameters of the discretization links are as follows: gain links - proportional link, integrator and differential link - 0,3s.

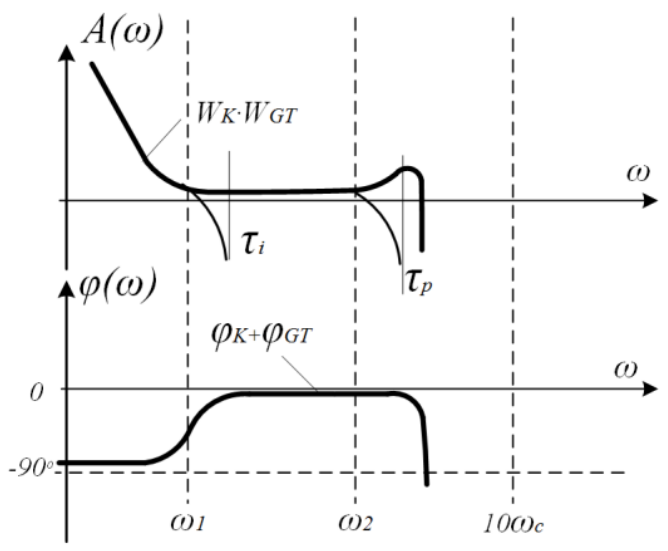

(a)
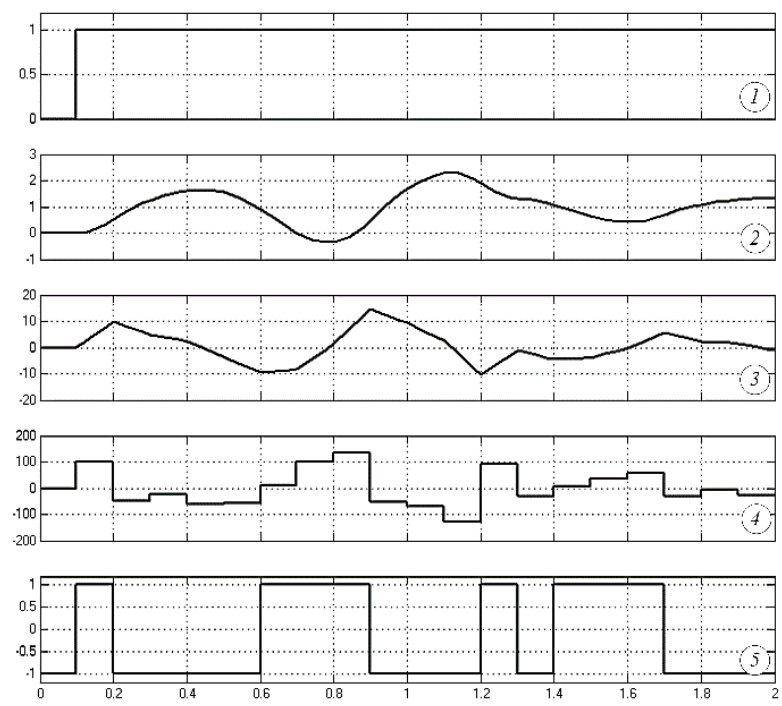

(b)

Figure 11.

Thus, the simulation has confirmed that the suppression link with the proposed frequency response "fits" very well into the frequency condition for the existence of slip and allows you to set different effective discreteness in the regulator channels.

Sample rate requirements might look like this:

$$
\tau_{i} \leq K T_{i} ; \quad \tau_{p} \leq \frac{T_{d}}{K} ; \quad \tau_{d} \leq \frac{1}{10 \omega_{c}} .
$$

The integral link in the amplification channel practically does not complicate the system. As shown in Figures 9-11, the frequency response of the amplifying link is practically not distorted by the integrator sampler, and the frequency response of the trajectory shaper by the proportional channel sampler in this link is shown in Figures 9-11. That is, as in the case of the PID controller, when the links are connected in parallel, fast sampling is needed for the link "operating" in the high-frequency region. 
For VSS with sliding processes, high-speed links are needed in the differential channel of the slip driver, and in the proportional channel of the gain link, while the integrator is responsible for accuracy, and can have many bits and a slow sampling rate, as in the case of the PID controller.

It should be assumed that the complication of the control object - an increase in the order of links or the presence of nonlinear structures will not complicate the analysis of slip conditions based on the frequency characteristics of the links of the system. That is, there is reason to believe that when creating sliding processes in a complex ACS, multifrequency sampling is possible. This assumption requires an undoubtedly independent study.

From the time and nature of the processes, it can be seen that the sliding processes are preserved at the speed of the channel for the formation of the slip function, which is determined in turn by the speed of the differential channel. Speed gain channels are not required, but precision is required. In this case, the slip condition is violated at high frequencies. This means that fast movements are not ideal, which is consistent with the model. This confirms the validity of the previously derived criteria for sliding along the frequency response and the effectiveness of the proposed frequency response suppression links for assessing the dynamics with nonlinear control.

In the VSS in which the conditions of stability (existence of sliding processes) are rather complicated even for linear ACS, the introduction of frequency characteristics of suppression links allows not only to obtain engineering slip conditions even for systems with nonlinear links, but also to optimize the resources of regulators according to the same principles of multifrequency sampling ", As ACS with PID controllers, possibly even with greater efficiency than in continuous ACS. This is due to the fact that in continuous systems with parallel connection of the links, each of them continues to work even where it is "not needed". For example, in a PID controller, the integrator works in the entire frequency range, its effect is weakened at high frequencies, but it can manifest itself negatively.

The presence of a sampling link more severely restricts the operation of channels, excluding these parasitic influences.

\section{Conclusions}

The proposed interpretation of the sampling links by the frequency characteristics of the suppression link makes it possible to formulate engineering stability conditions for digital systems, similarly to other stability criteria in frequency characteristics - the Nyquist criterion for linear control systems or the V.M. Popov for nonlinear ACS. Moreover, multifrequency sampling does not complicate the analysis process.

Simulation of ACS with multifrequency sampling confirmed the correctness of its main provisions and confirmed that the discreteness of signals in time in modern ACS is not only a minor factor, but it is a tool for building complex correction systems.

The proposed suppression link shows how you can use sampling resources to improve the efficiency of various channels of regulators responsible for accuracy and stability - amplification and stabilization channels.

The materials of the article prove that modeling processes in modern ACS is an effective and visual way of proving engineering recommendations based on provisions with a loose analytical interpretation.

\section{References}

1. Meerov M.V. Fundamentals of automatic control / M.V. Meerov, Yu.N. Mikhailov and V.G. Fridman / Moscow, "Nedra", - 1972.

2. Tsypkin I.Z. Fundamentals of the theory of automatic systems / Moscow, "Nauka", - 1977.

3. Voronov A.A. Fundamentals of the theory of automatic control: Automatic regulation of continuous linear systems / Moscow, "Energy”, - 1980, - 312 p.

4. Emelyanov S.V. New types of feedback: Management under uncertainty / Emelyanov S.V., Korovin S.K. // M.: "Science. Fizmatlit", 1997, - 352 p. 
5. Rush N. Direct Lyapunov method in the theory of stability / N. Rush, P. Abets, M. Lalua // - M.:"Mir", - 1980, $-300 \mathrm{p}$.

6. Kodkin V.L. The suppression link, its frequency properties / V.L. Kodkin, R.Kh. Gafiyatullin and E.R. Khaibyakov / Proceedings of the 4th International Conference on Automated Electric Drives / Magnitogorsk, Russia, September 14-17, 2004.

7. Kodkin V.L. Application of frequency characteristics of the suppression link in engineering calculations / V.L. Kodkin, R.Kh. Gafiyatullin, E.R. / Proceedings of the 4th International Conference on Automated Electric Drive / Magnitogorsk, Russia, September 14-17, 2004.

8. Kodkin V.L. Methods of optimizing the speed and accuracy complex guidance systems based on equivalence of automatic control system domain of attraction and unconditional stability of their equivalent circuits / Vestnik SUSU. Series "Computer technology, management, electronics", - 2017, - T.1, No. 1, - p. $23-33$.

9. Popov V.M. Hyper-stability of automatic systems / - Moscow: “Nauka”, 1970, - 456 p.

10. Sánchez, E.N., \& Ornelas-Tellez, F. (2013). Discrete-Time Inverse Optimal Control for Nonlinear Systems.DOI:10.1201/b14779

11. Almobaied M. A new inverse optimal control method for discrete-time systems. / M. Almobaied, I. Eksin and M. Güzelkaya /2015 12th International Conference on Informatics in Control, Automation and Robotics (ICINCO), DOI:10.5220/0005562902750280.

12. Almobaied M. Inverse Optimal Controller Design Based on Multi-Objective Optimization Criteria for Discrete-Time Nonlinear Systems / M. Almobaied, I. Eksin and M. Guzelkaya /2019 IEEE 7th Palestinian International Conference on Electrical and Computer Engineering (PICECE), DOI:10.1109/PICECE.2019.8747189.

13. Ornelas-Tellez F. Discrete-Time Nonlinear Systems Inverse Optimal Control: A Control Lyaponov Function Approach / F. Ornelas-Tellez, E.N. Sanchez and A.G. Loukianov / IEEE International Conference on Control Applications (CCA) Part of 2011 IEEE Multi-conference on Systems and Control, 2011.

14. Kipka R. The Discrete-Time Geometric Maximum Principle. / R. Kipka, R.R. Gupta / SIAM J. Control and Optimization, pp.2939-2961, DOI:10.1137/16M1101489.

15. Zhang, M., Gan, M., Chen, J., \& Jiang, Z. (2017). Adaptive dynamic programming and optimal stabilization for linear systems with time-varying uncertainty. 2017 11th Asian Control Conference (ASCC), pp.1228-1233. DOI:10.1109/ASCC.2017.8287346

16. Zhou, B. On asymptotic stability of linear time-varying systems. / Automatica, pp.266-276, DOI:10.1016 /j.automatica.2015.12.030.

17. Zhou B. On Asymptotic Stability of Discrete-Time Linear Time-Varying Systems. / B. Zhou, and T. Zhao / IEEE Transactions on Automatic Control, pp. 4274-4281. DOI:10.1109/tac.2017.2689499.

18. Kodkin V.L. Discreteness in Time and Evaluation of the Effectiveness of Automatic Control Systems: Examples of the Effect of Discreteness on Mathematical Patter / DOI: 10.5772/ intechopen. 91467. In book: Control Theory in Engineering. Publisher: IntechOpen. 